- they were mostly registered in the children's organized groups $(86,2 \%)$ including $12,0 \%$ cases in childcare facilities;

- they occurred more frequent in autumn-winter $(46,2 \%)$ and spring $(38,4 \%)$ periods of a year and were associated with consumption of vegetable salads in $72,4 \%$ cases;

- violation of sanitary-and-hygienic mode and technology of meal preparation from uncooked vegetables was noted in all outbreaks;

- laboratory confirmation of the diagnosis was set in $39,5 \%$ of the patients by PCR assay in the first days; bacteriological and serological diagnosis was confirmed in 14,1 and $64,6 \%$ after $2-3$ weeks;

- Yersinia pseudotuberculosis was revealed in the population of synanthropic and wild small mammals in $52,9 \%$, in transmission factor - in $46,7 \%$ from the total number of the studied outbreaks;

- all epidemic Y. pseudotuberculosis strains were O:1b serotype, possessed ypm gene of super-antigen, lacked of a high pathogenicity island (HPI), and belonged by plasmid content to single plasmid (pYV $47 \mathrm{MDa}$ ) and two-plasmid (pYV $47 \mathrm{MDa}$ and pVM $82 \mathrm{MDa}$ ) strains with the identical frequencies;

- the peculiarity of clinical manifestation of pseudotuberculosis caused by $Y$. pseudotuberculosis with two-plasmid and chromosomal tcpY1 gene (phagocytosis inhibitor) was the presence of the intoxication symptoms, fever, rash, damage of gastrointestinal tract, liver and joints with prevalence of medium-severe and severe course specific for Far Eastern scarlet-like fever (FESF);

- we discovered one more form of FESF clinical course caused by $Y$. pseudotuberculosis with $p Y V$ plasmid and lacking $t c p Y 1$ gene. In this case all observed symptoms were poorly expressed, and pseudotuberculosis was developed in "minor" easier form mainly in children.

The revealed peculiarities of pseudotuberculosis outbreaks are necessary to take into consideration in epidemiological surveillance.

\section{INTRASPECIFIC DIVERSITY OF YERSINIA PESTIS} CHAPERONE/USHER SECRETION APPARATUSES

S.V. Dentovskaya, L.A. Kadnikova, A.A. Kislichkina, A.G. Bogun, A.P. Anisimov

State Research Center for Applied Microbiology and Biotechnology, Obolensk, Russia

The Post-Antibiotic Era requires replacement of antibiotics with alternative antibacterials aimed at alternative molecular targets. One of such alternative approaches to treat infections are remedies targeting virulence. Yersinia pestis as many other Gram-negative bacterial pathogens use the chaperone/usher (CU) pathway to assemble virulence-associated surface fibers termed pili or fimbriae. Y. pestis has two well-characterized CU operons: the caf genes coding for the F1 capsule and the $p s a$ genes coding for the $\mathrm{pH} 6$ antigen. There are eight additional CU secretion systems capable of assembling $Y$. pestis pilus fibers. When choosing new targets for effective treatment of infectious diseases, it is necessary to search for pathogenicity factors possessing structural conservatism, since polymorphism gives pathogens the opportunity to evade interaction with the drug.

Searches and comparisons of amino acid sequences of $\mathrm{CU}$ proteins from $Y$. pestis strains belonging to SNPtypes 0.PE2, 0.PE3, 0.PE7, 0.PE4, 0.PE5, 1.ORI, 1.ANT, 2.ANT, and 2.MED were conducted using the databases of the National Center for Biotechnology Information (http://www.ncbi.nlm.nih.gov) by MAUVE (http://darlinglab.org), BLAST (https://blast.ncbi.nlm.nih.gov), ProtParam tool (https://web.expasy.org/protparam), and protein sequence analysis (http://molbiol.ru/scripts/01_18. html). The usher genes for two of chaperone/usher pathways (y1539-1544 and y4060-4063) were disrupted in all of the studied $Y$. pestis strains by an insertion sequence or premature stop codon, and thus these pathways are not expected to be functional. The phylogenetic-group-specific polymorphisms of amino acid sequences of the proteins from the $Y$. pestis $\mathrm{CU}$ secretion systems is inherent in five ushers $(y 0562, y 1858, y 1871, y 2390, y 3480)$, three molecular chaperone $(y 2392, y 3479$, caf1M) and three adhesin subunits (caf1, y2388, y3478). These polymorphic proteins are excluded from the list of potential $Y$. pestis molecular targets

This research was supported by the Russian Science Foundation (grant 14-15-00599).

\section{5 doi: 10.15789/2220-7619-2018-4-5.5 \\ THE OUTER MEMBRANE PROTEIN A (ompA) OF YERSINIA PESTIS IS NOT REQUIRED FOR VIRULENCE IN MICE AND RATS}

S.V. Dentovskaya, T.I. Kombarova, S.A. Ivanov,

T.E. Svetoch, R.Z. Shaikhutdinova

State Research Center for Applied Microbiology and Biotechnology, Obolensk, Russia

The plague bacterium Yersinia pestis has a number of well-described strategies to protect itself from the both cellular and humoral factors of the host's innate immunity. OmpA in several pathogens has been shown to mediate resistance to complement and antibacterial peptides, as well as play a role in invasion and intracellular survival. In this study, we sought to determine whether deletion of the $o m p A$ would render fully virulent $Y$. pestis strain attenuated in the mouse and rat models of plague.

$Y$. pestis $\triangle o m p A$ mutant was constructed using the knockout mutagenesis. SDS-PAGE and Western blot analyses with anti-OmpA serum showed the absence of OmpA in $Y$. pestis $\triangle o m p A$ cell lysates and outer membranes preparations. We could not detect any differences between $Y$. pestis wild type strains and their $\triangle o m p A$ derivatives using a serum killing assay. The OmpA deficient mutants were 2 times less resistant to bactericidal action of polymyxin B as compared with the wild type strains. To assess the biological significance of OmpA in fully virulent $Y$. pestis strain in vivo, studies in a mouse and rat models of bubonic and pneumonic plague were performed. Inbred mice and rats were infected subcutaneously or intranasally to mimic bubonic or pneumonic plague and observed for 21 days. Comparative study of the virulence of $Y$. pestis mutant strains using subcutaneously and intranasally challenged mice and intranasally challenged rats did not reveal differences in their $\mathrm{LD}_{50}$. The average survival time of mice and rats that succumbed to infection with the strain 231 or its isogenic derivative did not differ from each other. The estimated $\mathrm{LD}_{50}$ of the ompA mutant for subcutaneously challenged rats was approximately 10 -fold higher than the $\mathrm{LD}_{50}$ of the wild type 231 strain.

The main outcome of our investigation is the finding that the loss of the ability to produce OmpA antigen did not influence virulence of $\triangle o m p A$ mutant of $Y$. pestis. This argues against the usefulness of using OmpA as a molecular target for plague prophylaxis and therapy.

This study was supported by the Sectoral Scientific Program of the Russian Federal Service for Surveillance on Consumer Rights

Protection and Human Wellbeing. 